Pacific Journal of Mathematics

ON A QUESTION OF TARSI AND A MAXIMAL THEOREM 


\title{
ON A QUESTION OF TARSKI AND A MAXIMAL THEOREM OF KUREPA
}

\author{
J. D. HALPERN
}

\begin{abstract}
Let $P I$ be the statement, "Every Boolean algebra has a prime ideal."; let $S P I$ be the statement, "Every infinite set algebra has a nonprincipal prime ideal."; let $K$ be the statement, "Every family of sets includes a maximal subfamily of pairwise incomparable (by the inclusion relation) sets.". A model is exhibited of set theory without regularity in which $S P I$ and $K$ hold, $P I$ fails. Furthermore all finitary versions of the axiom of choice fail in this model and the model contains a set which is infinite Dedekind finite in the sense of the model. A proof is given that the local version of $S P I$ implies the axiom of choice for families of finite sets in $Z F$.
\end{abstract}

1. Introduction and statement of results. Before 1963, the only method available for constructing interesting models of ZermeloFraenkel set theory, $(Z F)$, was the Fraenkel-Mostowski-Specker method which produced models in which the axiom of regularity failed. In 1963, Cohen presented his method for producing models in which regularity holds. Questions concerning the axiom of choice, $(A C)$, can be considered in both types of models, unlike questions such as the continuum hypothesis which are only interesting in Cohen models. Although an independence result established by the Cohen method is stronger than the same result established by the FMS method, this latter method remains of interest for a number of reasons as Lévy points out in [2]. Two such reasons are as follows: (1) There are independence results which hold in set theory without regularity but do not hold in set theory with regularity. (2) The proof of a given independence may be more easily established by means of an FMS model. Such a proof may in turn give a clue to the stronger result. In this connection David Pincus has recently indicated a method of converting F'MS models to Cohen models which gives the stronger result in many cases. In this paper we will give an example of each eventuality mentioned above. Consider the following maximal principles:

PI. Every Boolean algebra has a prime ideal.

$S P I$. Every infinite set algebra has a nonprincipal prime ideal.

$K$. (Kurepa's principle). Every family of sets includes a maximal subfamily of pairwise incomparable sets. (Two sets are comparable if one is a subset of the other.)

We shall exhibit an FMS model in which $K$ holds but $C_{2}$ (the 
axiom of choice restricted to families whose elements are pairs) does not. On the other hand, Ulrich Felgner, [1], has shown that $K$, (actually, an equivalent of $K$ called Anti $K$ ) together with regularity implies $A C$. Hence we have an example of (1) above. In pre-Cohen days, some logicians felt that $A C$ might be indeed a consequence of regularity. As we see now, this feeling was incorrect as stated, but not so far off as one might think, since $K$ is a very weak maximal principal by one scale (in the absence of regularity it doesn't imply $C_{2}$ or for that matter $C_{n}$ for any natural number $n$ ). Also it does not exclude the existence of infinite Dedekind finite sets.

$S P I$ also holds in the above model. Since $P I \rightarrow C_{2}$ we obtain the independence result $S P I \nrightarrow P I$ in the absence of regularity, thus answering a question of Tarski, [7]. However using a method of David Pincus we are able to obtain a Cohen model in which $S P I \nrightarrow P I$, thus answering Tarski's question in full. This stronger result will appear in a subsequent paper. Although the original FMS proof of this result did give a clue to the proof of the stronger result, natural ways of converting it to a Cohen model proof did not work. New insight was required which gives rise to anew and conceptually more simple FMS proof. We shall also sketch this new $F M S$ proof.

In $\S 3$ we prove, using the axiom of regularity, that what Felgner [1] would call the local version of $S P I$ (actually we state an apparently weaker principle) implies the axiom of choice for families of finite sets. SPI-local and our version are as follows:

Definition. A set $X$ is surveyable if there is a choice function on $P_{<\omega}(X) . \quad\left(P_{<\omega}(X)\right.$ is the set of finite subsets of $X$ and a choice function for any set $Y$ is a function which assigns to every nonempty $y \in Y$, a member of $y$.)

Surveyable is a condition analogous to orderable and wellorderable. Clearly well-orderable $\rightarrow$ orderable $\rightarrow$ surveyable. Well known results of Mostowski and Läuchli establish that the converses are each false.

SPI-local: For any family of infinite sets there is a function which maps each member, $Y$, of the family to a nonprincipal prime ideal in $P(Y) . \quad(P(Y)$ is the power set of $Y$.)

$S P I-l s$ : ( $l$ is for "local" and $s$ is for "surveyable".) The same as SPI-local except that "infinite" is replaced by "infinite and surveyable".

One reason for the interest in $S P I$ is the result of Dana Scott (see [7]) that $S P I^{*} \leftrightarrow P I$ where $S P I^{*}$ is "Any proper ideal in a set algebra is included in a prime ideal." Note that $S P I$ is equivalent to: "The ideal of all finite sets in any infinite set algebra is included 
in a prime ideal." It is known that $P I$-local and hence $S P I^{*}$-local is equivalent to $P I$. Our results show that $S P I$-local is not equivalent to $S P I$ and leave open the question: "Does $S P I$-local imply PI?"

An earlier version of this paper contained the claim that SPIlocal is true in the model $M$ of $\S 2$. We are indebted to Paul Howard for pointing out this error and for other comments which led to the investigations concerning SPI-ls.

2. The Fraenkel-Mostowski model, M. Let $\mathscr{F}$ denote the set theory whose axioms are those of $Z F$ with regularity and $A C$ excluded. ${ }^{2}$ A transitive model of $\mathscr{F}$ or any subtheory of $\mathscr{F}$ is a model whose universe is a transitive set and whose membership relation is the usual membership relation restricted to its universe. We will identify a transitive model with its universe. An element in the universe of the model will be called a set of the model. A supercomplete transitive model is a transitive model satisfying the property that any subset of an element of the model is also an element of the model i.e. $x \in$ $M \rightarrow P(x) \subseteq M$. Existence of a supercomplete model of $\mathscr{F}+A C$ is implied by the existence of a strongly inaccessible cardinal. We start with a supercomplete transitive model $M_{1}$ of $\mathscr{F}+A C$ which has an element $S$ such that:

(1) Every element of $S$ is reflexive ( $x$ is reflexive if $x=\{x\}$ ).

(2) Any nonempty set $x$ of $M_{1}$ contains an element $y$ such that $y \cap x=0$ or $y \cap x \in S$.

(3) $S$ is denumerably infinite. ${ }^{3}$

Let $R$ be the inductively defined function on the ordinals in $M_{1}$, such that

$$
\begin{aligned}
& R(0)=S \\
& R(\beta)=\bigcup_{\lambda<\beta} P(R(\lambda))(P \text { denotes the power set }) .
\end{aligned}
$$

From the assumptions on $M_{1}$, one easily shows that any set of $M_{1}$ is a member of $R(\beta)$ for some ordinal $\beta$ in $M_{1}$. Let $G$ be the group of all automorphisms of $M_{1}$, i.e. $G$ consists of all permutations $\phi$ of $M_{1}$ such that $x \in y \leftrightarrow \phi(x) \in \phi(y)$ for any $x, y$ in $M_{1}$. Using the above characterization of $M_{1}$ one easily verifies that any permutation $\phi$ of $S$ induces a

1 We are indebted to the referee for suggesting that these remarks, and some others, be included in the paper.

2 We assume that our underlying (intuitive) universe of sets satisfies the axioms of $F+A C$ and, furthermore, that there exists an infinite set of reflexive sets ( $x$ is reflexive if $x=\{x\}$.) These assumptions make for a neater presentation but they are not necessary to achieve our independence results. The ideas presented here can be converted, easily, to the usual finitary proofs of relative consistency or to model theoretic proofs in the framework of the more familiar intuitive set theory where all sets are regular. Such conversion would use the results of [6].

3 Given our intuitive assumptions the existence of $M_{1}$ is also a consequence of the existence of a strongly inaccessible cardinal. 
unique automorphism of $M_{1}$ coinciding with $\phi$ on $S$.

Let the variable $e$ with or without subscripts range over finite subsets of $S$. Let $G[e]=\{\phi \in G: \phi$ leaves $e$ pointwise fixed $\}$. If $\phi(x)=$ $x$ for all $\phi \in G[e]$, we say that $x$ is e-symmetric and $e$ is a support of $x$. $x$ is finitely symmetric if it is $e$-symmetric for some $e$. Let $M=\{x \in$ $M_{1}: x$ and every member of the transitive closure of $x$ is finitely symmetric\}. $\quad M$ is obviously a transitive set. Furthermore one can use the arguments in [4] to show that $M$ is a model of $\mathscr{F}$; hence $M$ is a transitive model of $F$. It is easy to verify that $S$ is a Dedekind finite set in $M$.

The following facts about $M$ will prove useful:

1. If $x$ is finitely symmetric and $x \subseteq M$ then $x \in M$.

2. If $x$ is finite and $x \subseteq M$ then $x \in M$.

3. $M$ is closed under $G$. $G$ is a group of automorphisms of $M$. If $f$ is an $n$-ary operation on $M$ definable in the theory $\mathscr{F}$ then

$$
\phi\left(f\left(x_{1}, \cdots, x_{n}\right)\right)=f\left(\phi x_{1}, \cdots, \phi x_{n}\right) \text { all } \phi \in G, x_{1}, \cdots, x_{n} \in M .
$$

4. If $x \in M_{1}$ and the transitive closure of $x$ has empty intersection with $S$ then $x \in M$. In particular all constructible sets in $M_{1}$ are in $M$.

We will call the definition of a predicate or operation absolute if given any transitive model and sequence of elements in the model, the definition is satisfied by the sequence in the model if and only if it is satisfied by the sequence in the universe. A predicate or function will be called absolute if its usual definition is absolute. This notion of absolute is intended to be the semantic analogue of the notion given in [5] Pg.265. The notions probably differ but the difference will not appear in this paper or in any of the existing work on models of set theory. In particular most of the definitions in the development of general set theory are absolute (in both senses). The power set operation is a notable exception. For a list more than sufficient for this paper, see [5] Pgs. 267-269. In addition we note that $P_{s t} x=$ $P x \cap M$ for $x \in M$. (Where $P_{M}$ denotes the power set operation in the model $M$.)

Using these remarks it is not difficult to show that the truth of $S P I$ in $M$ is equivalent to:

$S P I_{M}$ : If $X \in M$ is an infinite set, there is a subset $I$ of $P X$ with the following properties

(0) $I \in M$.

(i) $I$ contains as members all finite subsets of $X$.

(ii) $I$ is closed under finite union.

(iii) For every $y \in P_{M} X, y \in I \leftrightarrow X-y \notin I$.

The truth of $K$ in $M$ is equivalent to:

$K_{M}$ : If $F \in M$, there is a maximal set among those sets $y \leqq F$ with the properties 


\section{(0) $M(y)$}

(i) Members of $y$ are pairwise incomparable. The falsity of $C_{n}$ in $M$ is equivalent to $\left(\neg C_{n}\right)_{M}$ : There exists $y \in M$, whose elements are disjoint $n$-sets and there is no $Z \in M$ whose intersection with each element of $Y$ is a singleten. We proceed to prove $S P I_{M}, K_{M},\left(\neg C_{n}\right)_{M}$.

Lemma 1. $G\left[e_{1} \cap e_{2}\right]$ is the smallest subgroup of $G$ including $G\left[e_{1}\right] \cup$ $G\left[e_{2}\right]$.

Proof. Clearly $G\left[e_{1}\right], G\left[e_{2}\right]$ are both included in $G\left[e_{1} \cap e_{2}\right]$. It remains to prove that $G\left[e_{1} \cap e_{2}\right]$ is included in any subgroup which includes $G\left[e_{1}\right] \cup G\left[e_{2}\right]$. Let $\phi \in G\left[e_{1} \cap e_{2}\right]$. Let $e_{1}-e_{2}=\left\{a_{1}, \cdots, a_{k}\right\}$ and let $c_{1}, \cdots, c_{k}$ be distinct elements not in $e_{1} \cup \phi^{-1}\left(e_{2}\right) \cup e_{2}$. Let $\phi_{1} \in G$ such that $\dot{\phi}_{1}$ permutes $a_{i}$ with $c_{i}$ for $i<k$ and is constant elsewhere on $S$. Then $\phi_{1} \in G\left[e_{2}\right]$. Let $e_{2}-e_{1}=\left\{b_{1}, \cdots, b_{p}\right\}$. Since $\dot{\phi}_{1} \phi^{-1}\left(b_{i}\right) \notin e_{1}$ and $b_{i} \notin e_{1}$ for $i<p$, there is $\phi_{2} \in G$ such that $\phi_{2}\left(b_{i}\right)=\dot{\phi}_{1} \phi^{-1}\left(b_{i}\right)$ for $i<p$ and $\phi_{2} \in G\left[e_{1}\right]$. Let $\dot{\phi}_{3}=\phi \phi_{1}^{-1} \phi_{2}$. Then $\phi_{3}\left(b_{i}\right)=\phi \phi_{1}^{-1} \phi_{2}\left(b_{i}\right)=\phi \phi_{1}^{-1} \phi_{1} \phi^{-1}\left(b_{i}\right)=$ $b_{i}$. Also $\phi_{3} \in G\left[e_{1} \cap e_{2}\right]$. Thus $\phi_{3} \in G\left[e_{2}\right]$. But $\phi=\dot{\phi}_{3} \dot{\phi}_{2}^{-1} \phi_{1}$, thus $\phi$ is any subgroup including $G\left[e_{1}\right] \cup G\left[e_{2}\right]$.

COROLLARY 2. The intersection over the set of supports of a set $x$ is a support of $x$.

Proof. Since supports are finite, the given intersection is also the intersection over some finite nonempty subset of the set of supports. It follows from Lemma 1 that the intersection of two supports of $x$ is again a support of $x$ since any element in the subgroup generated by $G\left[e_{1}\right] \cup G\left[e_{2}\right]$ can be represented as the composition of a finite number of automorphisms each of which pointwise fixes $e_{1}$ or $e_{2}$ and hence fixes $x$. With a trivial induction one obtains the same result for any finite number of supports.

Definition. We call the smallest support of $x$, the support of $x$ and denote it by $F(x)$.

Lemma 3. If $\dot{\phi}, \psi \in G$ and $\dot{\phi}|F(x)=\psi| F(x)$ then $\dot{\phi}(x)=\psi(x)$.

Proof. By hypothesis $\phi^{-1} \psi$ fixes $F(x)$ and hence $\phi^{-1} \psi^{\prime}(x)=x$, i.e. $\psi(x)=\phi(x)$.

Lemma 4. If $\dot{\phi} \in G$ and $\dot{\phi}(x)=x$, then $\phi(F(x))=F(x)$.

Proof. Let $\psi$ be any automorphism pointwise fixing $\phi(F(x))$. Then $\psi \phi(x)=\phi(x)=x$ by Lemma 3 and the second assumption. Thus $\phi(F(x))$ 
is a support of $x$ and hence $F(x) \subseteq \phi(F(x))$. Since $F(x)$ is finite this can only happen if $\phi(F(x))=F(x)$.

Note. $\dot{\phi}$ need not pointwise fix $F(x)$.

Lemma 5. If $\phi \in G$, then $\phi(F(x))=F(\phi(x))$.

Proof. First we note that if $\psi$ pointwise fixes $\phi(F(x))$ then $\psi \dot{\phi}(x)=$ $\phi(x)$ by Lemma 3. Hence $\phi(F(x)) \supseteqq F(\phi(x))$. Since this inclusion holds for all $x$ and $\phi$ we have $\phi^{-1} F(\phi(x)) \supseteqq F(x)$ i.e., $F(\phi(x)) \supseteqq \phi(F(x))$ which completes the proof.

In preparation for the proof of $S P I_{M}$, let us consider a fixed infinite set $X$ of $M$. For each $y \in X$, let

$$
\begin{aligned}
& f(y)=F(y)-F(X) \\
& T(y)=\{z: \exists \phi[\phi \in G[F(X)] \wedge \phi(y)=z]\} .
\end{aligned}
$$

$T(y)$ is the transitivity class of $y$ under $G[F(X)]$. Using Lemma 5 one easily shows

$$
\phi \in G[F(X)] \rightarrow \phi(f(y))=f(\phi(y)) .
$$

The following fact is the basic idea for the proof of $S P I_{M}$.

$$
\begin{aligned}
& \text { Let } y \in X \text { and }|T(y)|>1 \text {. } \\
& \text { Let } Q(w) \leftrightarrow \exists A \text {, finite } \forall z[z \in T(y) \cap w \rightarrow A \cap f(z) \neq 0] \text {. }
\end{aligned}
$$$$
\text { If } w \in P_{M}(X) \text {, then exactly one of } Q(w), Q(X-w) \text { hold. }
$$

Proof. Suppose neither hold. Then we obtain $z \in T(y) \cap w, t \in$ $T(y) \cap(X-w)$ such that $f(z) \cap F(w)=f(t) \cap F(w)=0$. Since $z, t \in$ $T(y)$, there exists $\phi \in G[F(X)]$ such that $\phi(z)=t$. Since $f(z), f(t)$ are disjoint from $F(X)$, there exists $\psi \in G[F(X)] \cap G[F(w)]$ such that $\psi|f(z)=\phi| f(z)$. By Lemma 3 we have $\psi(z)=\phi(z)=t$. Since $\psi$ pointwise fixes $F(w)$, we conclude $t \in w$ which is a contradiction.

Suppose both $Q(w)$ and $Q(X-w)$ hold. Let $A_{1}, A_{2}$ be the finite sets whose existence is asserted by $Q(w)$ and $Q(X-w)$ respectively. Let $\phi \in G[F(X)]$ map $f(y)$ onto $S-\left(A_{1} \cup A_{2}\right)$. Since $\phi(f(y))=f(\dot{\phi}(y))$ by (1), we have $f(\phi(y)) \cap\left(A_{1} \cup A_{2}\right)=0$. But $\phi(y) \in T(y) \cap X=(T(y) \cap w) \cup$ $(T(y) \cap(X-w))$ is a contradiction. This completes the proof of (2).

Proof of $S P I_{M}$. We consider two cases:

Case 1. There exists $y_{0} \in X$ such that $T\left(y_{0}\right)$ is infinite. Case 2. $T(y)$ is finite for every $y \in X$. 
For Case 1 we use (2) to obtain the desired result. No maximal principle is used here. For Case 2 the result follows directly from the fact that $S P I$ is true in $M_{1}$.

Proof of Case 1. Let $I$ consist of all $w \in P_{M}(X)$ such that $Q(w)$. We first show that $I$ is closed under $G[F(X)]$. If $\phi \in G[F(X)]$ and $w \in$ $I$, then $\phi(w) \subseteq X$ since $\phi$ is an automorphism neutral on $X$ and $\phi(w) \in$ $M$ since $M$ is closed under $G$. Furthermore $\phi\left(T\left(y_{0}\right) \cap w\right)=T\left(y_{0}\right) \cap \phi(w)$ since $T\left(y_{0}\right)$ is closed under $G[F(X)]$ and $\phi$ is an automorphism. Since $Q(w)$ holds we have an $A$ such that

$$
z \in T\left(y_{0}\right) \cap w \rightarrow A \cap f(z) \neq 0 .
$$

Thus $\phi(z) \in T\left(y_{0}\right) \cap \phi(w) \rightarrow \phi(A) \cap f(\phi(z)) \neq 0$, using the automorphism property of $\phi$ and (1). But any element of $T(y) \cap \phi(w)$ is $\phi(z)$ for some $z \in T(y) \cap w$. Thus $\phi(w) \in I$ and $I$ is closed under $G[F(X)]$. Hence $I$ is finitely symmetric and since $I \subseteq M$ we have

$$
I \in M \text {. }
$$

If $w$ is finite and $w \subseteq X$, then $w \in M$. Also $Q(w)$ because $\cup\{f(z): z \in$ $w\}$ is the required finite $A$. Thus

(i) $I$ contains as members all finite subsets of $X$. If $w, z \in I$, then $w \cup z \in P_{M}(X)$ and the required $A$ to establish $Q(w \cup z)$ is just the union of the two sets which establish $Q(w), Q(z)$ respectively. Thus,

(ii) $I$ is closed under finite union. We obtain directly from (2) that

(iii) for every $y \in P_{M} X, y \in I \leftrightarrow X-y \notin I$. This completes the proof of case 1 .

Case 2. Suppose $T(y)$ is finite for every $y \in X$. Then $F(y) \subseteq F(X)$. For suppose on the contrary that there exists $a \in F(y)-F(X)$. Then for each $b \in S-F(X)$ there exists $\phi \in G[F(X)]$ such that $\phi(a)=b$. From this fact and Lemma 4 one easily concludes the contradiction that $T(y)$ is infinite, Thus $F^{\prime}(y) \subseteq F^{\prime}(X)$. From the definition of $T^{\prime}(y)$ we see that actually $T(y)$ contains only the element $y$. However the important point is that there is a support which supports every element of $X$. This is a very strong condition on $X$. In particular it implies that any subset of $P(X)$ belongs to $M$. With the aid of $S P I$, we obtain a nonprincipal prime ideal $I$, in the algebra of sets $P X$. By the previous remarks $I \in M$.

Proof of $K_{M}$. Let $X \in M$. Then the set $Z=\{Y: Y \subseteq X \wedge Y \in$ $M \wedge$ members of $Y$ are pairwise incomparable $\wedge F(Y) \subseteq F(X)\}$ has a 
maximal element: Using the fact that every element of $Z$ is supported by $F(X)$ we establish that the union over a chain in $Z$ is a member of $Z$. $Z$ is nonempty since $0 \in Z$. With the aid of Zorn's form of $A C$, we conclude that $Z$ has a maximal element $Y_{0}$.

We proceed to show that $Y_{0}$ is the desired maximal set. Suppose not. Then there exists $y \in X-Y_{0}$ such that $Y_{0} \cup\{y\}$ consists of incomparable sets. Let $Y_{1}=Y_{0} \cup\{\phi(y): \phi \in G[F(X)]\}$. Then since $Y_{0} \subseteq$ $Y_{1} \subseteq X$ and $Y_{1} \in M$ and $F\left(Y_{1}\right) \subseteq F(X)$ and $Y_{0}$ is maximal in $Z$, we conclude that $Y_{1}$ includes two comparable elements. We have two cases.

Case 1. There is $z \in Y_{0}$ and $\phi \in G[F(X)]$ such that $z \subseteq \phi(y)$ or $\phi(y) \subseteq z$.

Case 2. There are $\dot{\phi}_{1}, \phi_{2} \in G[F(X)]$ such that $\phi_{1}(y) \neq \phi_{2}(y)$ and $\phi_{1}(y) \subseteq \phi_{2}(y)$.

If Case 1 holds we have $\phi^{-1}(z) \leqq y$ or $y \leqq \phi^{-1}(z)$. Since $\phi^{-1}(z) \in Y_{0}$, this contradicts $Y_{0} \cup\{y\}$ consists of incomparable sets.

If Case 2 holds, we have $y \leqq \phi(y)$ and $y \neq \phi(y)$ for $\dot{\phi}=\dot{\phi}_{1}^{-1} \dot{\phi}_{2}$. Let

$$
\begin{aligned}
& A_{1}=\left\{w \in F(y): \exists n\left[n>0 \wedge \phi^{n}(w)=w\right]\right\} \\
& A_{2}=\left\{z \in S: \exists w \exists n\left[w \in A_{1}, \wedge \phi^{n}(w)=z\right]\right\} .
\end{aligned}
$$

$A_{2}$ is finite since $A_{1}$ is finite and every element of $A_{1}$ has only finitely many images under successive iteration of $\phi$. Also $A_{2}$ is closed under $\phi$. Thus $\phi \mid A_{2}$ generates a cyclic group which is a group of permutations of a finite set, and thus has finite order say $n_{0}$.

If $z \in F(y)-A_{1}$, there is a natural number $n_{z}$, such that if $m>n_{z}$, $\phi^{m}(z) \notin F(y)$. This assertion follows from the finiteness of $F(y)$ and the fact that for each $n>0, \phi^{n}(z) \neq z$.

Let $m=$ the first multiple of $n_{0}$ greater than $\max \left\{n_{z}: z \in F(y)-\right.$ $\left.A_{1}\right\}$. Then $\phi^{m}(w)=w$ if $w \in A_{2}$ and, hence, if $w \in A_{1}$. Also, $\phi^{m}(z) \notin$ $F(y)$ if $z \in F(y)-A_{1}$. Thus if $z \in F(y) \cap\left\{\phi^{m}(w): w \in F(y)\right\}$ then $\phi^{m}(z)=$ $z$. Hence there is a permutation $\psi$ of $S$ such that

(a) $\psi(z)=\phi^{m}(z)$ for $z \in F(y)$

(b) $\psi\left(\phi^{m}(z)\right)=z$ for $z \in F(y)$.

From (a) we obtain

(c) $\psi(y)=\phi^{m}(y)$ since $\psi^{-1} \phi^{m}$ is the identity on $F(y)$. From (b) we obtain

(d) $\psi \phi^{m}(y)=y$.

By hypothesis $y \subseteq \phi(y)$ and since $\phi$ is an automorphism we have

(e) $y \leqq \phi(y) \cdots \subseteq \phi^{m}(y)$.

Thus by (c), $y \leqq \psi(y)$. But (e) also yields $\psi(y) \subseteq \psi \dot{\phi}^{m}(y)$. Applying $(\mathrm{d})$, we have $\psi(y) \subseteq y$. Thus $y=\psi(y)$. From (c) we deduce $y=\phi^{m}(y)$. 
But this together with (e) yields the contradiction $y=\phi(y)$.

Proof of $\left(\neg C_{n}\right)_{M}$ for $n>0$ :

Let $Y=\{\{(X, x): x \in X\}: X \subseteq S \wedge|X|=n\} . \quad Y \in M$ since $M$ is a model of $\mathscr{F}$ and the relations and operations used in its definition are absolute and the fact that $x$ finite and $x \leqq y \in M \Rightarrow x \in M$. Also $Y$ consists of disjoint sets of cardinality $n$. Suppose $Z$ is a choice set for $Y$ and $Z \in M$. Since $S$ is infinite and $F(Z)$ is finite we can find $X \subseteq S,|X|=$ $n$, and $X \cap Z=0$. Let $\phi \in G[F(Z)]$ such that $\phi(X)=X$ and $\phi(x) \neq x$ all $x \in X$. Then if $(X, x) \in Z,(X, \phi(x)) \in Z$ and $x \neq \phi(x)$, contradicting $Z$ is a choice set for $Y$.

The new proof of $S P I \rightarrow P I$ to which allusion was made in the introduction comes about as follows:

(1) It is easy to verify that if for every countably infinite $Y$, $P(Y)$ has a nonprinciple prime ideal then for every Dedekind infinite $X, P(X)$ has a nonprincipal prime ideal. (If $X$ is Dedekind infinite and $Y \subseteq X$ is countably infinite and $I$ is a nonprincipal prime ideal in $P(Y)$ then $\{Z: Z \subseteq X \& Z \cap Y \in I\}$ is a nonprincipal prime ideal in $P(X)$.)

(2) If $M$ is any $F M S$ model and $Y \in M$ and $Y$ is well-orderable within $M$ (i.e. in the $M$-sense) then $P_{M}(Y)=P(Y)$ and $P_{M} P_{M}(Y)=$ $P(P(Y))$.

Since we assume $A C$ holds in the universe it follows from (1) \& (2) that if $M$ is an FMS model in which every nonfinite set of the model is Dedekind infinite in the $M$-sense then $S P I$ holds in the model. In [3] Azriel Levy considers a whole class of such models. It is easy to verify that $C_{2}$ fails in these models. Hence any one of these models establishes our independence $S P I \leftrightarrow P I .^{4}$

Actually this argument establishes the independence (SPI \& [Dedekind infinite $=$ infinite] $\nrightarrow C_{2}$ ). In light of this argument the following stronger version of the original result is interesting: $(S P I \nrightarrow$ [infinite $=$ Dedekind infinite] or $\left.C_{2}\right) .^{4}$

3. $S P I-l s \rightarrow C_{<\omega}$.

Let $P(X)$ denote the power set of $X$. Let $P_{n}(X)$ denote the set of all $n$ element subsets of $X$, for any natural number $n$, and let $P_{<\omega}(X)$ denote the set of all finite subsets of $X$. We prove the following theorem which is apparently stronger than the title of this section since it omits the "nonprinciple".

${ }_{4}$ The referee has informed us that this proof was discovered independently by Norman Noble. 
THeOREM. If for every set $X$ there is a function which assigns to every infinite surveyable set $Y \leqq X$ a prime ideal in $P(Y)$, then for every set $X$ there is a choice function for $P_{<\omega}(X)$.

A function such as the one hypothesized for $X$ in the theorem will be called a $P I$ function for $X$. A disjointed set is one whose elements are pairwise disjoint.

Lemma 1. Given $f$ a choice function on $P_{<\omega}(X)$ and $g, a P I$ function for $X$, one can define a choice function $h$ on the set of finite disjointed subsets of $P(X)$.

Proof. Let $Y$ be a finite disjointed subset of $P(X)$.

Case 1. $\cup Y$ is finite. Then $\cup Y \in P_{<\omega}(X)$. Since $Y$ is disjointed we can define $h(Y)=$ unique $y \in Y$ such that $f(\cup Y) \in y$.

Case 2. $\cup Y$ is infinite. Then there is exactly one $y \in Y$ such that $y \notin g(\cup Y)$. Define $h(Y)$ to be this unique $y$.

Lemma 2. Given $h$ a choice function on the set of finite disjointed subsets of $P(X)$ one can define a choice function $k$ on $P_{<\omega}(P(X))$.

Proof. We shall define $k$ on $P_{n}(P(X))$ by recursion on $n$. For $u \in P_{1}(P(x))$ we define $k(u)=h(u)$. Suppose $n>1$ and $u \in P_{n}(P(X))$. Let $w=\{z: z$ is an atom in the subalgebra of $P(X)$ generated by $u \wedge z \neq \cap u \wedge z \neq 0 \wedge z \neq \cap\{X-x: x \in u\}\}$. $w$ is a finite disjointed subset of $P(X)$ and since $|u|>1, w$ is nonempty. Let $u_{1}=\{x \in u: f(w) \subseteq$ $x\}, u_{1} \neq 0$ since $f(w) \in w$ and thus is included in some element of $u$. $\left|u_{1}\right|<n$ since $f(w) \in w$ and thus is included in the complement of some element in $u$ and thus (since $f(w) \neq 0) f(w)$ is not included in this element of $u$. So we can define $k(u)=k\left(u_{1}\right)$.

Proof of the theorem. Let $\tau$ be the 1-place term of Zermelo Fraenkel set theory defined by ordinal recursion as follows:

$$
\tau(\alpha)=P\left(\bigcup_{\beta<\alpha} \tau(\beta)\right) \text {. }
$$

It follows from the axiom of regularity that $\forall x \exists \alpha[x \subseteq \tau(\alpha)]$. We shall prove the theorem by showing that for each ordinal $\alpha$ there is a function $g$ such that for all $\beta<\alpha, g(\beta)$ is a choice function on $P_{<\omega}(\tau(\beta))$. We define $g$ by ordinal recursion up to $\alpha$. Let $f$ be a $P I$ function for $\tau(\alpha)$. Suppose, for each $\gamma<\beta, g(\gamma)$ is a choice function on $P_{<\omega}(\tau(\gamma))$ such that $g\left(\gamma_{1}\right) \leqq g\left(\gamma_{2}\right)$ if $\gamma_{1} \leqq \gamma_{2}$. Then $\bigcup_{r<\beta} g(\gamma)$ is a choice function on $P_{<\omega}\left(\mathbf{U}_{r<\beta} \tau(\gamma)\right)$. Since $\beta<\alpha, \mathbf{U}_{r<\beta} \tau(\gamma) \cong \tau(\alpha)$. Hence $f$ is a $P I$ function for $\bigcup_{r<\beta} \tau(\gamma)$. Applications of Lemmas 1 and 2 allow us to define a 
choice function on $P_{<\omega}(\tau(\beta))$. Call this function $g^{\prime}$. In order to satisfy the recursion condition we define

$$
g(\beta)=\left(g^{\prime}\left[P_{<\omega}(\tau(\beta))-\bigcup_{\gamma<\beta} P_{<\omega}(\tau(\gamma))\right]\right) \cup \bigcup_{\gamma<\beta} g(\gamma) .
$$

\section{REFERENCES}

1. Ulrich Felgner, Die existenz wohlgeordneter konfinaler Tielmengen in Ketten und das Auswahlaxiom, Math. z., 111 (1969), 221-232.

2. Azriel Lévy, The Fraenkel-Mostowski method for independence proofs in set theory, Symposium on the Theory of Models, North-Holland (1965) 221-228.

3. - The interdependence of certain consequences of the axiom of choice, Fund. Math., 54 (1964), 135-157.

4. A. Mostowski, Uber die Unabhängigkeit des Wohlordnungssatz vom Ordnungsprinzip, Fund. Math., 32 (1939), 201-252.

5. J. R. Shoenfield, Mathematical Logic, Addison-Wesley Publishing Co. 1967.

6. E. Specker, Zur Axiomatic der Mengenlehre (Fundierungs und Auswahlaxiom), Zeitschrift f. Math. Logik und Grundlagen d. Math., 3 (1957), 173-210.

7. A. Tarski, Prime ideal theorems for set algebras and the axiom of choice, B.A.M.S. 60 (1954), 391.

Received October 5, 1970 and in revised form September 9, 1971. The preparation of this papar was supported in part by a grant from the National Science Foundation.

The UNIVERSity of TOLEDo 



\section{PACIFIC JOURNAL OF MATHEMATICS}

\section{EDITORS}

H. SAMELSON

Stanford University

Stanford, California 94305

C. R. Hовву

University of Washington

Seattle, Washington 98105
J. DugundJI

Department of Mathematics

University of Southern California

Los Angeles, California 90007

RICHARD ARENS

University of California

Los Angeles, California 90024

\section{ASSOCIATE EDITORS}
E. F. BECKENBACH
B. H. NeumanN
F. WOLF
K. YOSHIDA

\section{SUPPORTING INSTITUTIONS}

UNIVERSITY OF BRITISH COLUMBIA

CALIFORNIA INSTITUTE OF TECHNOLOGY

UNIVERSITY OF CALIFORNIA

MONTANA STATE UNIVERSITY

UNIVERSITY OF NEVADA

NEW MEXICO STATE UNIVERSITY

OREGON STATE UNIVERSITY

UNIVERSITY OF OREGON

OSAKA UNIVERSITY
UNIVERSITY OF SOUTHERN CALIFORNIA

STANFORD UNIVERSITY

UNIVERSITY OF TOKYO

UNIVERSITY OF UTAH

WASHINGTON STATE UNIVERSITY

UNIVERSITY OF WASHINGTON

$\stackrel{*}{*} \stackrel{*}{*} \stackrel{*}{*}{ }^{*}$ AMERICAN MATHEMATICAL SOCIETY

NAVAL WEAPONS CENTER 


\section{Pacific Journal of Mathematics}

\section{Vol. 41, No. $1 \quad$ November, 1972}

Anatole Beck and Peter Warren, Weak orthogonality.................

Jonnie Bee Bednar and Howard E. Lacey, Concerning Banach spaces whose duals are abstract $L$-spaces.............................

Louis Harvey Blake, Canonical extensions of measures and the extension of regularity of conditional probabilities..........................

R. A. Brooks, Conditional expectations associated with stochastic processes ..........................................

Theodore Allen Burton and Ronald Calvin Grimmer, On the asymptotic behavior of solutions of $x^{\prime \prime}+a(t) f(x)=e(t) \ldots \ldots \ldots \ldots \ldots \ldots$

Stephen LaVern Campbell, Operator-valued inner functions analytic on the closed disc ............................................

Yuen-Kwok Chan, A constructive study of measure theory...

Alexander Munro Davie and Bernt Karsten Oksendal, Peak interpolation sets for some algebras of analytic functions ...................

H. P. Dikshit, Absolute total-effective $\left(N, p_{n}\right)(c, 1)$ method ...............

Robert E. Edwards, Edwin Hewitt and Kenneth Allen Ross, Lacunarity for

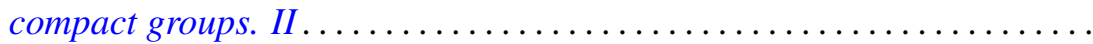

James Daniel Halpern, On a question of Tarski and a maximal theorem of Kurepa

Gerald L. Itzkowitz, A characterization of a class of uniform spaces that admit an invariant integral

Mo Tak Kiang, Semigroups with diminishing orbital diameters ..

Glenn Richard Luecke, A class of operators on Hilbert space ...

R. James Milgram, Group representations and the Adams spectral sequence. . .

G. S. Monk, On the endomorphism ring of an abelian p-group, and of a large subgroup...

Yasutoshi Nomura, Homology of a group extension ...

R. Michael Range, Approximation to bounded holomorphic functions on strictly pseudoconvex domains...

Norman R. Reilly, Inverse semigroups of partial transformations and $\theta$-classes.

Chris Rorres, Strong concentration of the spectra of self-adjoint operators

Saharon Shelah, A combinatorial problem; stability and order for models and theories in infinitary languages.

George Gustave Weill, Vector space decompositions and the abstract

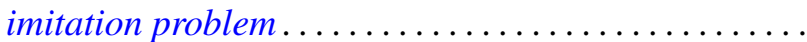

